\title{
A retrospective Comparison of Clinical Outcome Following Conventional In-vitro Fertilization (c-IVF) vs Intracytoplasmic Sperm Injection (ICSI) for $\mathbf{1 0 0 \%}$ Teratozoospermia Patients
}

\author{
Mingzhao Li, Xia Xue and Juanzi Shi* \\ ART Center, Northwest Women's and Children's Hospital, Xi'an 710003, PR China \\ *Corresponding author: Juanzi Shi, ART Center, Northwest Women's and Children's Hospital, Xi'an 710003, PR China, Tel: +8618191022131; E-mail: \\ szzxsjz@163.com
}

Received date: April 25, 2017; Accepted date: May 22, 2017; Published date: May 31, 2017

Citation: Li M, Xue X, Shi J (2017) A retrospective Comparison of Clinical Outcome Following Conventional In-Vitro Fertilization (c-IVF) vs Intracytoplasmic Sperm Injection (ICSI) for 100\% Teratozoospermia Patients. Reproductive Immunol Open Acc 2: 32. doi: 10.21767/2476-1974.100032

Copyright: (C) $2017 \mathrm{Li} \mathrm{M}$, et al. This is an open-access article distributed under the terms of the Creative Commons Attribution License, which permits unrestricted use, distribution, and reproduction in any medium, provided the original author and source are credited.

\section{Abstract}

Objectives: To investigate whether ICSI could improve the clinical outcomes of the $100 \%$ teratozoospermia patients.

Methods: This study contained 200 IVF-ET and 216 ICSI-ET cycles from January 2013 to January 2016. The first cycle patients aged $<40$ years, with cycle day $3 \mathrm{FSH}<10 \mathrm{mlU} / \mathrm{mL}$ and 8-12 antral follicles were included. Main outcome measure(s): clinical, obstetric and neonatal outcome.

Results: The characteristics of the patients showed no significant differences in the female's age, Gn administration, $\mathrm{Gn}$ does, basal serum follicle-stimulating hormone, basal E2 value, endometrial thickness, infertile time, the number of retrieved oocytes and the number of transferred embryos between two groups $(p>0.05)$. The proportion of blastocyst transfers was significantly higher in c-IVF group than ICSI group ( 41.00 versus $28.70 \%$; $p=0.008$ ). We observed that the implantation ( 53.80 versus $44.85 \%$; $\mathrm{p}=0.016)$ and clinical pregnancy (67.00 versus $60.19 \%$; $\mathrm{p}=0.149)$ rates were higher in c-IVF group than those in ICSI group. In addition, the abortion (7.46 versus $16.15 \%$; $p=0.028)$ and ectopic pregnancy (0.75 versus $5.38 \%$; $\mathrm{p}=0.028$ ) rates were significantly lower in $\mathrm{c}-\mathrm{IVF}$ group compared with ICSI group. The two groups showed no significant differences in obstetrical and neonatal outcomes ( $p>0.05)$.

Conclusions: For isolated $100 \%$ teratozoospermia patients, ICSI should not be considered to improve the clinical outcomes if the semen meet c-IVF standard in the first cycle.

Keywords: $100 \%$ Teratozoospermia; C- IVF; ICSI; Clinical outcomes

\section{Introduction}

Male factor infertility was necessary in almost half of couples receiving infertility treatment [1]. Regularly, couples with infertility treatment should carry out semen analysis including parameters as sperm count, motility and morphology. As known, single semen parameter could not accurately predict the successful pregnancy probability with assisted reproduction [2], but some studies indicated that the normal sperm morphology rate (NSMR) was associated with clinical outcomes $[3,4]$.

However, the predictability of low morphology by strict criteria of causing severe infertility was challenged [5]. Some subsequent studies failed to find strict morphology as a good prediction of male subfertility. More importantly, the reference value for NSMR recommended was <30\% in 1992, <14\% in 1999 and $<4 \%$ in 2010 which also suggested that NSMR might not be highly correlated with successful pregnancy. Meanwhile, the application of appropriate threshold became increasingly uncertain as a result of substantial increases in the reference values [6]. Shi et al. [7] showed that NSMR had some influence on IVF-ET, and 5\% NSMR exhibited a higher value than 4\% NSMR in predicting the outcomes of IVF. In addition, some studies had indicated that NSMR according to the criteria of WHO5 had but a limited value in predicting the outcomes and neonatal status following IVF-ET $[8,9]$.

In some patients, the motile sperms $(a+b)$ met the c-IVF standard but all the sperms were abnormal in the morphology. And it was not clear whether or not ICSI should be applied for these patients. With this in mind, we aimed to explore whether ICSI can improve the clinical outcomes of $100 \%$ teratozoospermia patients.

\section{Methods}

\section{Patients and setting}

This study contained 200 first IVF-ET and 216 first ICSI-ET cycles from January 2013 to January 2016. For c-IVF patients, all 
the semen samples were fresh semen. For ICSI patients, the frozen semen and the semen extracted by testicular sperm aspiration (TESA) were not contained. The rescue-ICSI patients were not included in this study. The inclusion criteria: female, aged $<40$ years, with cycle day $3 \mathrm{FSH}<10 \mathrm{mIU} / \mathrm{mL}$ and $8-12$ antral follicles. Male, the abnormality of spermatozoon occur in head. The exclusion criteria: female patients with endometrial fibroids and endometriosis were ruled out. Male patients with $Y$ chromosome deficiency or no motile sperm were ruled out. All the ICSI couples with enough motile sperm were included.

\section{Ovarian stimulation protocol}

For controlled ovarian hyperstimulation, the patients used the standard long protocols with GnRH agonist (GnRH-a, Decapeptyl, Germany) and recombinant FSH (GONAL-f, Merck, Serono, Italy; Puregon, Organon, Netherlands). Oocyte retrieval was performed $36 \mathrm{~h}$ later by transvaginal ultrasonography-guided aspiration.

\section{Semen analysis and fertilization}

The initial semen analysis was performed following the standard procedure [10]. Strict morphology was evaluated using the Kruger strict criteria. C-IVF fertilization is performed 2-2.5 $\mathrm{h}$ after oocytes retrieved. Fertilization was allowed to occur naturally and one oocyte was incubated with nearly 40000 sperm. Short term fertilization was adopted and the cumulus granule cells were peeled off 4-4.5 $\mathrm{h}$ after fertilization. If the number of sperm $(a+b)$ in the semen sample was $<10 \times 106$, then the prepared sperm suspension was used for ICSI. The oocytes were pre-cultured in the fertilization medium (IVF, Vitrolife, Sweden) with $10 \%$ protein supplement for 2-4 h before ICSI. Each oocyte was placed into $4 \mu \mathrm{L}$ droplets of IVF medium covered under warm mineral oil for ICSI [11].

\section{Embryo culture and grading}

Embryos were placed in a $50 \mu \mathrm{L}$ drop of cleavage medium (G1, Vitrolife, Sweden) supplemented with $5 \%$ protein supplement and covered with paraffin oil in a humidified atmosphere under $5 \% \mathrm{CO} 2$ at $37^{\circ} \mathrm{C}$ for prior $48 \mathrm{~h}$. On day 3 , the number of highquality embryos were more than four, they would be incubated to $\mathrm{G} 2$ culture medium (Vitrolife, Sweden) until D5 for transfer. Embryonic cleavage and morphologic appearance were assessed 64 to $68 \mathrm{~h}$ after fertilization. A morphologic score was given for day-3 embryo according to Li's previous studies [12].

A morphologic score was given for day-3 embryo according to the number of blastomeres, homogeneous degree of blastomeres and degree of cytoplasmic fragmentation: grade I (8-10 blastomeres, even homogeneous blastomeres $<10 \%$ cytoplasmic fragmentation), grade II (6-7 or $<10$ blastomeres with even homogeneous blastomeres of no cytoplasmic fragmentation, 8-10 blastomeres, even homogeneous blastomeres with $10 \%-20 \%$ cytoplasmic fragmentation), grade III (uneven and nonhomogeneous blastomeres with 20-50\% cytoplasmic fragmentation), and grade IV (uneven and nonhomogeneous blastomeres with $>50 \%$ cytoplasmic fragmentation). Grades I and II were identified as high-quality embryos. Clinical pregnancy was confirmed by the presence of a gestational sac.

\section{Statistical analysis}

Analysis was performed with SPSS for Windows (version 17.0, SPSS Inc., Chicago, IL, USA). The paired Student's t-test was applied for comparing means. The $\chi 2$-tests test was used for group comparison of rate. Statistical significance was defined when $\mathrm{p}<0.05$.

\section{Results}

Table 1 showed the clinical parameters between c-IVF group and ICSI group. We could see that there were no significant differences in the female's age, $G$ n administration, $G n$ does, basal serum follicle-stimulating hormone, basal E2 value, endometrial thickness, infertile time, the number of retrieved oocytes and the number of transferred embryos between two groups $(p>0.05)$. However, $c-I V F$ group shows a higher proportion of blastocyst transfers compared with ICSI group ( 41.00 versus $28.70 \% ; p=0.008$ ).

Table 1: General description of the individuals who participated in this study.

\begin{tabular}{|c|c|c|c|}
\hline \multirow{2}{*}{ Parameter } & \multicolumn{3}{|l|}{ NSMR=0\% } \\
\hline & C-IVF & ICSI & P Value \\
\hline Cycle number (n) & 200 & 216 & - \\
\hline Female age $(y)$ & $29.22 \pm 3.36$ & $\begin{array}{ll}28.81 & \pm \\
3.59 & \end{array}$ & 0.296 \\
\hline Gn administration (d) & $10.18 \pm 1.85$ & $\begin{array}{ll}10.05 & \pm \\
1.91 & \end{array}$ & 0.629 \\
\hline Gn does (IU) & $27.98 \pm 10.73$ & $\begin{array}{ll}28.05 & \pm \\
10.97 & \end{array}$ & 0.431 \\
\hline Basal serum FSH (IU/L) & $6.44 \pm 1.47$ & $6.58 \pm 1.49$ & 0.389 \\
\hline Basal serum E2 (pg/mL) & $39.08 \pm 29.51$ & $\begin{array}{ll}40.12 & \pm \\
30.43 & \end{array}$ & 0.217 \\
\hline Endometrial thickness (cm) & $11.39 \pm 2.52$ & $\begin{array}{ll}11.53 & \pm \\
2.69 & \end{array}$ & 0.312 \\
\hline Infertility duration $(\mathrm{y})$ & $3.83 \pm 2.45$ & $4.01 \pm 2.17$ & 0.297 \\
\hline Oocytes retrieved $(n)$ & $11.69 \pm 4.48$ & $\begin{array}{ll}11.96 & \pm \\
4.84\end{array}$ & 0.419 \\
\hline Embryo transferred (n) & $1.71 \pm 0.41$ & $1.80 \pm 0.43$ & 0.103 \\
\hline $\begin{array}{l}\text { Blastocyst transfer cycle } \\
\text { rate }(\%, n)\end{array}$ & $41.00(82 / 200)$ & $\begin{array}{l}28.70 \\
(62 / 216)\end{array}$ & 0.008 \\
\hline
\end{tabular}

Table 2 showed the clinical outcomes between c-IVF group and ICSI group. We observed that the normal fertilization $(62.87$ versus 68.44\%; $p=0.229)$, cleavage (98.32 versus 99.09\%; $p=0.052$ ), high-quality embryo (45.46 versus $46.97 \% ; p=0.380$ ) and twin pregnancy (36.57 versus $33.85 \% ; p=0.644$ ) rates were no significant differences between c-IVF group and ICSI group. The implantation ( 53.80 versus $44.85 \% ; p=0.016$ ) and clinical pregnancy $(67.00$ versus $60.19 \% ; p=0.149)$ rates were higher in c-IVF group than ICSI group. In addition, the abortion (7.46 
versus $16.15 \% ; p=0.028)$ and ectopic pregnancy ( 0.75 versus $5.38 \% ; p=0.028$ ) rate were significantly lower in $c-I V F$ group than
ICSI group. Moreover, c-IVF group showed a lower high-quality embryo rate than ICSI group (38.10 versus 46.97\%; $p=0.001$ ).

Table 2: Comparison of clinical outcomes for $100 \%$ teratozoospermia patients in different clinical assisted reproductive method.

\begin{tabular}{|c|c|c|c|}
\hline \multirow{2}{*}{ Parameter } & \multicolumn{3}{|l|}{ NSMR $=0 \%$} \\
\hline & c-IVF & ICSI & P Value \\
\hline Cycle number (n) & 200 & 216 & - \\
\hline Fertilization $(\%, n)$ & $81.52(1906 / 2338)$ & $71.62(1534 / 2142)$ & $<0.001$ \\
\hline Normal fertilization (d) & $62.87(1470 / 2338)$ & $68.44(1466 / 2142)$ & 0.229 \\
\hline Cleavage $(\%, n)$ & $98.32(1874 / 1906)$ & $99.09(1520 / 1534)$ & 0.052 \\
\hline High-quality embryo $(\%, n)$ & $45.46(852 / 1874)$ & $46.97(714 / 1520)$ & 0.38 \\
\hline Implantation (\%, n) & $53.80(184 / 342)$ & $44.85(174 / 388)$ & 0.016 \\
\hline Clinical pregnancy $(\%, n)$ & $67.00(134 / 200)$ & $60.19(130 / 216)$ & 0.149 \\
\hline Abortion $(\%, n)$ & $7.46(10 / 134)$ & $16.15(21 / 130)$ & 0.028 \\
\hline Twin-pregnancy $(\%, n)$ & $36.57(49 / 134)$ & $33.85(44 / 130)$ & 0.644 \\
\hline Ectopic pregnancy $(\%, n)$ & $0.75(1 / 134)$ & $5.38(7 / 130)$ & 0.028 \\
\hline
\end{tabular}

Table 3: Comparison of obstetrical and neonatal outcomes for $100 \%$ teratozoospermia patients in different clinical assisted reproductive method.

\begin{tabular}{|c|c|c|c|}
\hline \multirow{2}{*}{ Parameter } & \multicolumn{3}{|l|}{ NSMR=0\% } \\
\hline & C-IVF & ICSI & $P$ Value \\
\hline Cycle number (n) & 200 & 216 & - \\
\hline Live birth $(\%, \mathrm{n})$ & $52.00(104 / 200)$ & $46.30(100 / 216)$ & 0.245 \\
\hline Sex ratio (F/M) & $1.08(54 / 50)$ & $1.00(50 / 50)$ & 0.784 \\
\hline Birth weight (g, Twins) & $2805.27 \pm 416.59$ & $2799.35 \pm 438.36$ & 0.297 \\
\hline Birth weight (g, Singleton) & $3239.42 \pm 467.58$ & $3287.66 \pm 491.27$ & 0.478 \\
\hline Birth weight <2,500 g $(\%, n)$ & $3.85(4 / 104)$ & $3.00(3 / 100)$ & 0.74 \\
\hline Gestational age (weeks, Twins) & $38.62 \pm 1.51$ & $38.87 \pm 1.73$ & 0.399 \\
\hline Gestational age (weeks, Singleton) & $36.75 \pm 1.98$ & $36.54 \pm 1.79$ & 0.508 \\
\hline Prematurity <37 weeks $(\%, n)$ & $6.73(7 / 104)$ & $9.00(9 / 100)$ & 0.547 \\
\hline \multicolumn{4}{|l|}{ Mean Apgar score } \\
\hline $1 \mathrm{~min}$ & $9.05 \pm 0.21$ & $9.03 \pm 0.26$ & 0.62 \\
\hline $5 \mathrm{~min}$ & $9.83 \pm 0.33$ & $9.79 \pm 0.28$ & 0.479 \\
\hline $10 \mathrm{~min}$ & $9.95 \pm 0.38$ & $9.93 \pm 0.34$ & 0.548 \\
\hline \multicolumn{4}{|l|}{ Neonatal complications (n) } \\
\hline Pathological jaundice & 1 & 1 & - \\
\hline Low birth weight & 4 & 3 & - \\
\hline Respiratory problems & 2 & 0 & - \\
\hline Neonatal death (n) & 0 & $\begin{array}{l}2 \text { ( } 1 \text { for asphysia, } 1 \text { for congenital heart } \\
\text { disease) }\end{array}$ & - \\
\hline
\end{tabular}

Table 3 showed the obstetrical and neonatal outcomes between c-IVF group and ICSI group. In c-IVF group, the live birth rate was $52.00 \%$ which was no significant difference with that of ICSI group (46.30\%). In the c-IVF group there were 164 babies 
( 80 boys and 84 girls) and in the ICSI group there were 140 babies ( 70 boys and 70 girls).

In the c-IVF group, the mean birth weight of singleton and twins were $3239.42 \pm 467.58 \mathrm{~g}$ and $2805.27 \pm 416.59 \mathrm{~g}$, similar with that of ICSI group ( $p>0.05)$. The gestational age of singleton and twins were $38.62 \pm 1.51$ week and $36.75 \pm 1.98$ week in cIVF group which was also similar with that of ICSI group ( $p>0.05$ ). Mean Apgar scores (at 1, 5, and $10 \mathrm{~min}$ ) were also no significant differences between c-IVF group and ICSI group $(p>0.05)$. In $c$-IVF group, seven neonatal complications were observed, including one with pathological jaundice, four with low birth weight and 2 with respiratory problems. No neonatal death was found in c-IVF group. Four neonatal complications were observed in ICSI group, including one with pathological jaundice and three with low birth weight. Two neonatal deaths were found in ICSI group including one for asphysia and one for congenital heart disease.

\section{Discussion}

The definition of teratozoospermia in WHO is frequently revised in recent years. The reference values defined teratozoospermia had a substantial increase but the appropriate threshold to apply is uncertain $[13,14]$. Shi et al. [7] showed that NSMR had some influence on IVF-ET, and 5\% NSMR exhibited a higher value than $4 \%$ NSMR in predicting the outcomes of c-IVF. He et al. [13] observed that NSMR $\leq 4 \%$ affected the total rate of fertilization while NSMR $\leq 3 \%$ reduced the rate of normal fertilization in IVF. Therefore, the reference value for teratozoospermia still needs a more persuasive research. Besides, there is still no clear conclusion whether NSMR affect the clinical outcomes of c-IVF and ICSI.

Some studies have demonstrated that ICSI assisted reproduction have a higher fertilization rate compared with cIVF assisted reproduction for the patients with NSMR<4\% [11]. And in a large retrospective study for NSMR $<4 \%$ patients, $\mathrm{Li}$ et al. [11] observed that the abortion rate was significantly lower in ICSI group than that in IVF group (6.32 versus $13.6 \%$; $p<0.001$ ). These results suggested ICSI assisted reproduction might be a good choice for the teratozoospermia patients. The reason was that during the process of ICSI, the embryologist could choose individual sperm that appear morphologically "normal" from even the most abnormal specimens. This aim of this study was to explore the effects of different assisted reproductive methods (c-IVF/ICSI) on the clinical outcomes for $100 \%$ teratozoospermia patients. In addition, this paper presented a preliminary view for $100 \%$ teratozoospermia patients whose semen reach c-IVF standard, c-IVF should be given in priority and ICSI could not improve the clinical outcomes.

It is noted that, when ICSI performed, the implantation and pregnancy rates decreased, and the abortion and ectopic rates increased. For decreased implantation and pregnancy rates in ICSI assisted reproduction, reasons might be that, it showed a higher proportion of blastocyst transfers in c-IVF assisted reproduction. It was no doubt that the implantation and pregnancy rates after cleavage-stage embryo transfer was not as better as blastocyst stage embryo transfer $[15,16]$. Interestingly,
ICSI assisted reproduction had a similar good-quality rate with cIVF reproduction in our study. For $100 \%$ teratozoospermia patients, ICSI assisted reproduction might not affect the embryo development ability during the period of cleavage stage. However, ICSI assisted reproduction might make a negative effect on the process of extended culture to blastocyst stage. In recent studies, Ohgi et al. [17] also confirmed that the blastocyst formation rate was significantly lower in ICSI assisted reproduction compared with c-IVF assisted reproduction. Kihaile et al. [18] reported that despite ICSI oocytes had significantly higher fertilization rates than c-IVF oocytes, subsequent rates of development from cleavage stage to blastocyst stage were similar. These results suggested that ICSI assisted reproduction should be used with caution. Some studies had shown that the ectopic rate was significantly higher in ICSI assisted reproduction than that in C-IVF assisted reproduction due to fewer risk factors in c-IVF assisted reproduction [19]. We also observed that the abortion rate was improved in ICSI assisted reproduction which was not consistent with Li's study [11]. Previous studies had indicated that, for teratozoospermia patients, ICSI assisted reproduction could reduce the abortion rate. Our opposite conclusion might be that ICSI assisted reproduction had lost the advantage to rule out the sperm with morphological abnormalities for $100 \%$ teratozoospermia patients. Above all, we could conclude that both the embryo development and clinical outcomes were not improved by ICSI assisted reproduction.

In the comparison of obstetrical and neonatal outcomes between c-IVF and ICSI groups, we observed a higher birth rate in c-IVF group than ICSI group. Our results showed that the method of assisted reproduction (c-IVF/ICSI) was not related with sex ratio for $100 \%$ teratozoospermia patients. A recent study analyzed 27,158 singleton infants after fresh singleembryo transfer, which concluded that ICSI was associated with a significant reduction in sex ratio (M/F) [20]. In a study of 2014 from multi-center in China, they also obtained a similar conclusion, when ICSI was used sex ratio was imbalance toward females of $50.3 \%$ compared to $47.7 \%$ when IVF was performed $(p<0.01)[21]$.

It is vital to select an appropriate fertilization method to provide patients with the best chance of success. ICSI procedure may cause some damage to oocytes and not allow the oocyte to fuse with the best sperm via natural selection, but a large number of studies have confirmed that ICSI does not necessarily cause adverse effects upon obstetric and neonatal outcomes $[22,23]$. In our comparison of obstetric and neonatal outcomes, c-IVF and ICSI had no significant difference in the of birth weight, gestational age and Mean Apgar score. No neonatal death was found in c-IVF group. Two neonatal deaths were found in ICSI group including one for asphysia and one for congenital heart disease. There is no doubt that some different opinion existed in other researchers. Nouri et al. [24] concluded that the process of pregnancy is more complicated after IVF, but the fetal outcome seemed to be better in this group than ICSI group. Lie et al. [25,26] indicated that ICSI treatment shows more significant risks of major birth defects in addition to the risks included in IVF treatment. In our study, seven neonatal complications were observed in c-IVF group and four were observed in ICSI group. 
In summary, for $100 \%$ teratozoospermia patients, ICSI might not be considered to improve the clinical outcomes if the semen reach c-IVF standard in the first cycle. It still needs to accumulate more clinical data for a persuasive conclusion.

\section{References}

1. Aydos OS, Yükselten Y, Kaplan F (2015) Analysis of the correlation between sperm DNA integrity and conventional semen parameters in infertile men. Turk J Urol 41: 191-197.

2. Kruger TF, Coetzee K (1999) The role of sperm morphology in assisted reproduction. Hum Reprod Update 5: 172-178.

3. Kruger TF, Acosta AA, Simmons KF (1988) Predictive value of abnormal sperm morphology in in vitro fertilization. Fertil Steril 49: 112-7.

4. Grow DR, Oehninger S, Seltman HJ (1994) Sperm morphology as diagnosed by strict criteria: probing the impact of teratozoospermia on fertilization rate and pregnancy outcome in a large in vitro fertilization population. Fertil Steril 62: 559-567.

5. Check JH, Bollendorf A, Wilson C (2007) A retrospective comparison of pregnancy outcome following conventional oocyte insemination vs intracytoplasmic sperm injection for isolated abnormalities in sperm morphology using strict criteria. J Androl 28: 607-612.

6. Natali I, Muratori M, Sarli V (2013) Scoring human sperm morphology using Tests implets and Diff-Quik slides. Fertil Steri 99: 1227-1232.

7. Shi YC, Shen LY, Cheng HB (2014) Relationship of sperm morphology with the outcomes of in vitro fertilization and embryo transfer. Zhonghua Nan Ke Xue 20: 690-696.

8. Shu JH, Feng GX, Li J (2013) Predictive value of sperm morphology according to WHO Laboratory Manual for the Examination and Processing of Human Semen (5th Ed) on the outcomes of IVF-ET. Zhonghua Nan Ke Xue 19: 414-417.

9. Ghirelli-Filho M, Mizrahi FE, Pompeo AC (2012) Influence of strict sperm morphology on the results of classic in vitro fertilization. Int Braz J Urol 38: 519-528.

10. Ricci G, Perticarari S, Boscolo R (2009) Semen preparation methods and sperm apoptosis: swim-up versus gradient-density centrifugation technique. Fertil Steril 91: 632-638.

11. Li B, Ma YF, Huang J (2014) Probing the effect of human normal sperm morphologyrate on cycle outcomes and assisted reproductive methods selection. PLoS One 9: e113392.

12. Li M, Ma C, Xue X (2015) Effect of normal sperm morphology rate (NSMR) on clinical outcomes and fertilization methods selection in the ultra-short-term GnRH-a protocol. Gynecol Endocrinol 6: 1-4.

13. Auger J, Jouannet P, Eustache $F$ (2016) Another look at human sperm morphology. Hum Reprod 31: 10-23.
14. He B, Cheng JP, Pan Q (2016) Normal sperm morphology and the outcomes of routine in vitro fertilization. Zhonghua Nan Ke Xue 22: 32-36.

15. Fragouli E, Alfarawati S, Spath K (2014) Morphological and cytogenetic assessment of cleavage and blastocyst stage embryos. Mol Hum Reprod 20: 117-126.

16. Maheshwari A, Hamilton M, Bhattacharya S (2015) Should we be promoting embryo transfer at blastocyst stage? Reprod Biomed Online 1472: 6483-6485.

17. Gardner DK, Lane M, Stevens J (2000) Blastocyst score affects implantation and pregnancy outcome: towards a single blastocyst transfer. Fertil Steril 73: 1155-1158.

18. Ohgi S, Hagihara C, Anakubo H (2016) A comparison of the clinical outcomes of embryos derived from intracytoplasmic sperm injection after early fertilization check and conventional insemination using sibling oocytes. Arch Gynecol Obstet 293: 887-892.

19. Kihaile PE, Misumi J, Hirotsuru K (2003) Comparison of sibling oocyte outcomes after intracytoplasmic sperm injection and in vitro fertilization in severe teratozoospermic patients in the first cycle. Int J Androl 26: 57-62.

20. Li MZ, Zhao WQ, Ren AQ (2015) Association of fertilization strategy and embryo transfer time with the incidence of ectopic pregnancy. Zhonghua Nan Ke Xue 21: 913-916.

21. Arikawa M, Jwa SC, Kuwahara A (2016) Effect of semen quality on human sex ratio in in vitro fertilization and intracytoplasmic sperm injection: an analysis of 27,158 singleton infants born after fresh single-embryo transfer. Fertil Steril 105: 897-904.

22. Bu Z, Chen ZJ, Huang G (2014) Live birth sex ratio after in vitro fertilization and embryo transfer in China--an analysis of 121,247 babies from 18 centers. PLoS One 9: e113522.

23. Oron G, Fisch B, Sapir O (2014) Pregnancy outcome after ICSI with thawed testicular sperm from men with non-obstructive azoospermia compared to ICSI with ejaculated sperm from men with severe oligoasthenoteratozoospermia and IVF with normal ejaculated sperm. Gynecol Endocrinol 30: 103-106.

24. Wen J, Jiang J, Ding C (2012) Birth defects in children conceived by in vitro fertilization and intracytoplasmic sperm injection: a metaanalysis. Fertil Steril 97: 1331-1337.

25. Nouri K, Ott J, Stoegbauer L (2013) Obstetric and perinata outcomes in IVF versus ICSI-conceived pregnancies at a tertiary care center--a pilot study. Reprod Biol Endocrinol 11: 84

26. Lie RT, Lyngstadaas A, Ørstavik KH (2005) Birth defects in children conceived by ICSI compared with children conceived by other IVFmethods; a meta-analysis. Int J Epidemiol 34: 6. 